\title{
Autoimmune Pancreatitis Exhibiting Multiple Mass Lesions
}

\author{
Masahiro Shiokawa Yuzo Kodama Yukiko Hiramatsu \\ Akira Kurita Yugo Sawai Norimitsu Uza Tomohiro Watanabe \\ Tsutomu Chiba \\ Department of Gastroenterology and Hepatology, Graduate School of Medicine, \\ Kyoto University, Kyoto, Japan
}

\section{Key Words}

Autoimmune pancreatitis - Pancreatic cancer - Endoscopic ultrasound-guided fine needle aspiration $\cdot \lg G 4 \cdot$ Mass forming

\begin{abstract}
Our case is a first report of autoimmune pancreatitis with multiple masses within the pancreas which was pathologically diagnosed by endoscopic ultrasound-guided fine needle aspiration and treated by steroid. The masses disappeared by steroid therapy. Our case is informative to know that autoimmune pancreatitis sometimes exhibits multiple masses within the pancreas and to diagnose it without unnecessary surgery.
\end{abstract}

\section{Introduction}

Autoimmune pancreatitis (AIP) is a unique form of chronic pancreatitis associated with an autoimmune inflammatory process $[1,2]$. Although diffuse swelling of the pancreatic parenchyma and diffuse irregular narrowing of the pancreatic duct system are morphologically characteristic of AIP, a focal type of this clinical entity has been recently recognized [3]. The focal type of AIP exhibits a localized mass lesion in the pancreas, similar to pancreatic carcinoma [4]. Consequently, some patients with these features have been subjected to surgical exploration with a presumed diagnosis of pancreatic carcinoma. Considering that AIP shows a favorable response to steroid therapy, the differentiation of these two entities is clinically important to avoid surgery.

There have been only a few cases of AIP with multifocal lesions [5] but no case pathologically diagnosed by endoscopic ultrasound-guided fine needle aspiration (EUS-FNA) and treated by steroid. In this report, we describe the clinical, radiological and histopathological features of a patient with AIP who exhibited distinct double masses in the pancreas and was treated by steroid. 


\section{Case Report}

A 63-year-old male patient without any symptoms was admitted because of pancreatic masses that were picked up on a medical checkup. He had a history of diabetes mellitus but none of alcohol abuse. On physical examination, the patient showed bilateral swelling of the submandibular gland. On laboratory examination, his serum glucose was $179 \mathrm{mg} / \mathrm{dl}$ (normal range 65-109 mg/dl), $\gamma$-GTP was $132 \mathrm{IU} / \mathrm{l}$ (normal range 9-54.0 IU/l), cancer antigen $19-9$ level was $55.5 \mathrm{ng} / \mathrm{ml}$ (normal range 0-37.0 ng/ml) and serum IgG4 was $773 \mathrm{mg} / \mathrm{dl}$ (normal range $4.8-105 \mathrm{mg} / \mathrm{dl}$ ). Other serological tests, including pancreatic and hepatobiliary enzymes, $\gamma$-globulin, immunoglobulin $G$ and tumor markers (CEA and DUPAN-II) were within the normal range. Antinuclear antibodies were also negative at a titer of $<1: 20$.

On abdominal ultrasonography, the patient was found to have a low-echoic mass in the pancreatic head uncus and body. Contrast-enhanced computed tomography (CT) showed two mass lesions in the head and body of the pancreas (fig. 1a, b); the lesions were 18 and $10 \mathrm{~mm}$ in diameter, respectively. The tumors showed slight attenuation in the delayed phase (fig. 1c, d). On dynamic magnetic resonance imaging, they were also hypovascular during the early phase and showed delayed enhancement during the late phase. Endoscopic retrograde cholangiopancreatography showed localized narrowing of the main pancreatic duct at the pancreatic head. The common bile duct was not dilated. Fluorine-18 fluorodeoxyglucose positron emission tomography (FDG-PET) showed FDG uptake in the bilateral submandibular glands, hilar, mediastinal lymph nodes and pancreatic head uncus and no uptake in the body of the pancreas.

EUS-FNA of the two masses and normal pancreas was performed and the specimens were adequate for cytology and cell block. Cytology in the two masses was rich in inflammatory cells. The cell block in the two masses revealed a dense lymphoplasmacytic infiltrate and significant replacement of pancreatic parenchyma by irregular fibrosis (fig. 2a). Immunostaining for IgG4 revealed diffuse infiltrate of IgG4-positive plasma cells (fig. 2b). Cytology and the cell block in the normal pancreas had no abnormality (fig. 2c).

On the basis of the above-mentioned clinical, imaging, and cytological findings, a diagnosis of AIP was made. The patient was treated with prednisone, which was initiated at a dose of $30 \mathrm{mg} /$ day with a tapering schedule of $5 \mathrm{mg}$ every 2 weeks. One month after treatment, the elevated IgG level resolved and CT revealed that the two masses had disappeared (fig. 3), and FDG uptake in the pancreas, submandibular glands, hilar and mediastinal lymph nodes had disappeared completely.

\section{Discussion}

We show the clinical, radiological, histopathological and clinical features of a patient with AIP who had distinct double masses in the pancreas and steroid therapy. Since Yoshida et al. [2] proposed the term 'autoimmune pancreatitis' in 1995, this unique pancreatic disorder was recognized as a new clinical entity with such characteristics as increased serum $\gamma$-globulin, IgG levels, the presence of autoantibody, diffuse enlargement of the parenchyma, and irregular narrowing of the pancreatic duct $[1,6]$. In 2001, Hamano et al. reported that patients with AIP have a high serum concentration of IgG4 [7]. The specificity and sensitivity of a high serum IgG4 level in the diagnosis of AIP are $>90 \%$ and this is now believed to be the most useful examination for diagnosing AIP. Hirano et al. [8] recently reported eight cases of AIP with locally affected lesions. They found that the localized type of AIP is more difficult to differentiate from pancreatic cancer radiologically because typical radiological features reflecting diffuse involvement are not observed. They reported that serum IgG4 was elevated in the cases of focal AIP and that serum IgG4 levels might be helpful for its diagnosis. However, a pancreatic cancer with elevated serum IgG4 was reported [9]. 
Multiple masses in the pancreas are a rare clinical entity. As previously reported, multiple pancreatic masses were as follows; metastatic pancreatic tumors such as renal cell carcinoma [10], pancreatic neuroendocrine tumor in multiple endocrine neoplasia type 1 [11], pancreatic cancer [12], and AIP [5, 13]. Our patient had no primary lesion other than pancreas and the imaging findings in the present case were not suitable for pancreatic neuroendocrine tumor, but pancreatic cancer could not be excluded based on the radiological images. Radiological findings were indicative of a malignant pancreatic tumor, especially pancreatic cancer. Also, few reports on the coexistence of AIP and pancreatic ductal adenocarcinoma have been published, posing a new problem in the management of AIP $[14,15]$.

EUS-FNA of the pancreas or EUS trucut biopsy (TCB) are useful for diagnosing AIP and excluding pancreatic cancer [16]. FNA in this patient was compatible with AIP. However, pancreatic cancer is difficult to exclude by biopsy specimens alone because of reactive inflammatory cell reactions and fibrosis surrounding the neoplastic cells.

It is interesting in the present case that multiple masses had formed in the pancreas. These multiple lesions have been rarely reported for AIP until now. We could not explain this unusual manifestation, but the early phase of AIP could be focal lesions. The most important thing is that we recognized that AIP can manifest as multiple mass lesions similar to those in the present case, and thus one should examine IgG4, perform EUS-FNA or TCB-FNA and try to exclude pancreatic cancer. If IgG4 is elevated and EUS-FNA excludes malignancy, steroid therapy should be carefully challenged and the response quickly check by CT or PET, so that the patient may avoid unnecessary surgery. This case is a first report of AIP with multifocal masses treated by steroid and we believe this diagnostic process is informative. 

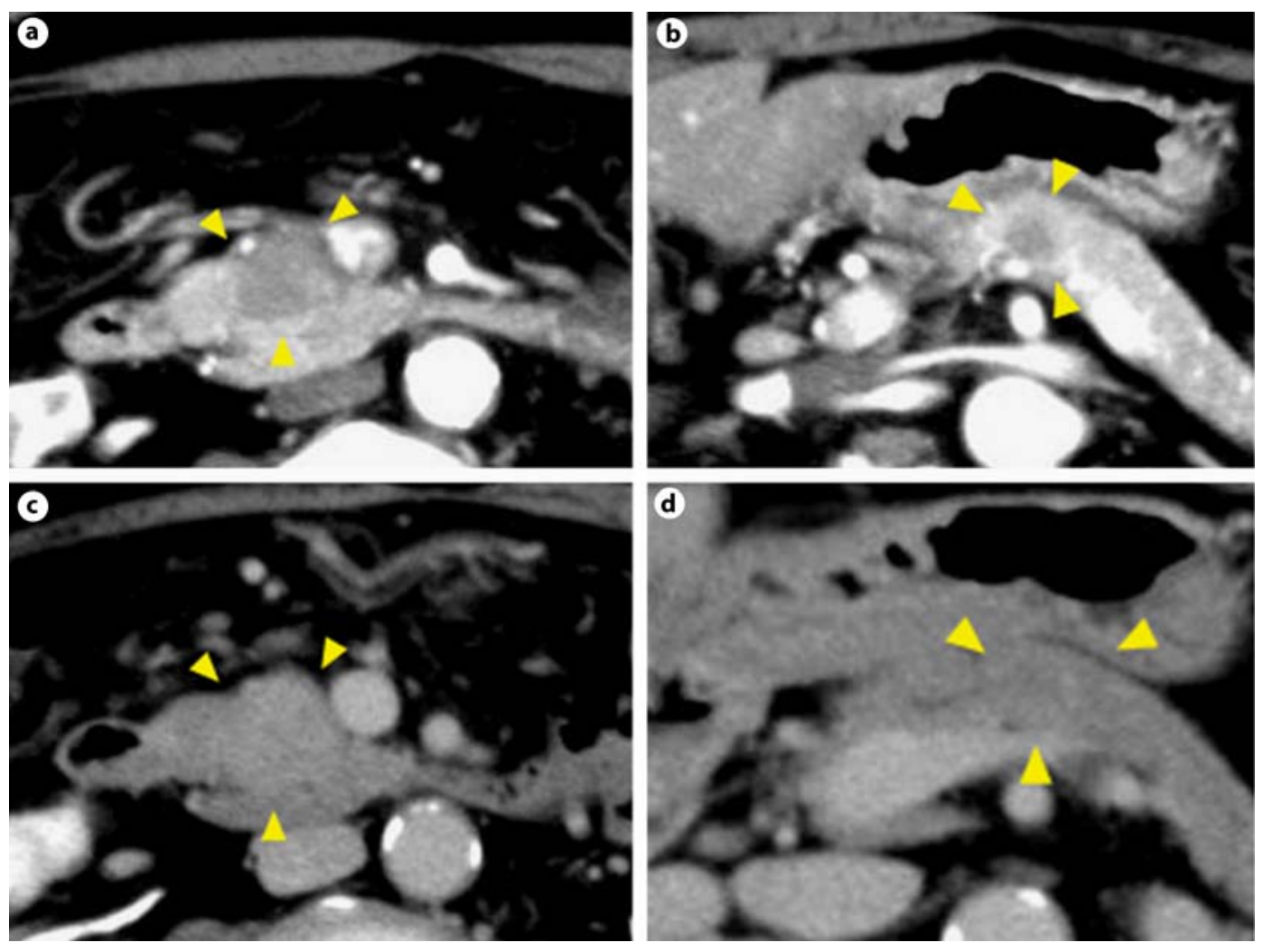

Fig. 1. CT revealed mass lesions in the head (a) and body (b) of the pancreas (arrows); early phase. They showed slight delayed enhancement during the late phase (arrows in $\mathbf{c}, \mathbf{d}$ ). 

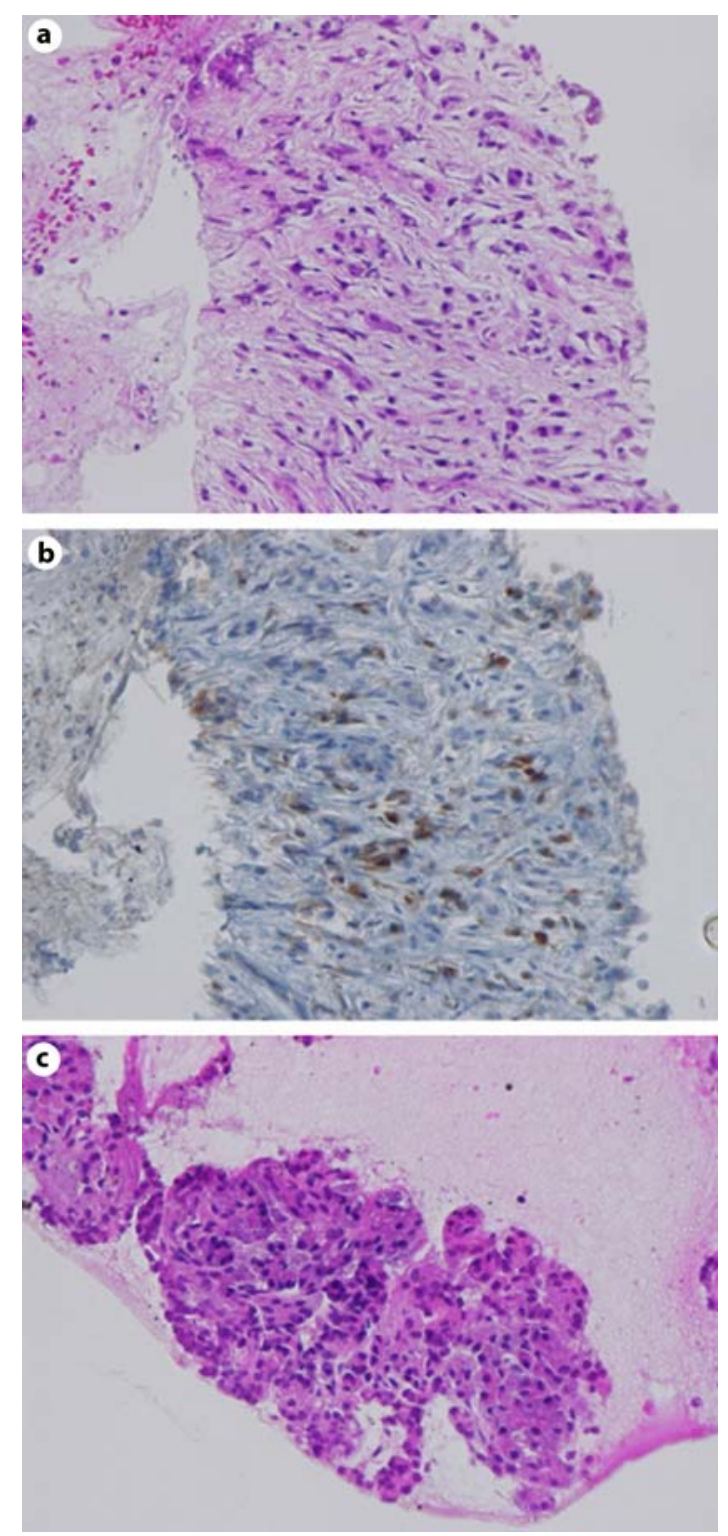

Fig. 2. Cell block in the two nodules showed dense lymphoplasmacytic infiltrate and significant replacement of pancreatic parenchyma by irregular fibrosis $(H \& E, \times 400)(\mathbf{a})$. Numerous plasma cells in the two nodules showed positive immunoreactivity for IgG4 $(\mathrm{H} \& \mathrm{E}, \times 400)(\mathbf{b})$. Cell block in the normal pancreas showed almost normal acinar cells, no lymphoplasmacytic infiltrate and no fibrosis $(\mathrm{H} \& \mathrm{E}, \times 400)(\mathbf{c})$. 


\begin{tabular}{r|l|l|l}
$\begin{array}{r}\text { Case Reports in } \\
\text { Gastroenterology }\end{array}$ & $\begin{array}{l}\text { Case Rep Gastroenterol 2011;5:528-533 } \\
\text { Dol: 10.1159/000331799 }\end{array}$ & $\begin{array}{l}\text { Published online: } \\
\text { September 10, 2011 }\end{array}$ & $\begin{array}{l}\odot \text { ISSN 1662-0631 } \\
\text { ISS } 1 \text { arger AG, Basel } \\
\text { www.karger.com/crg }\end{array}$ \\
\hline
\end{tabular}
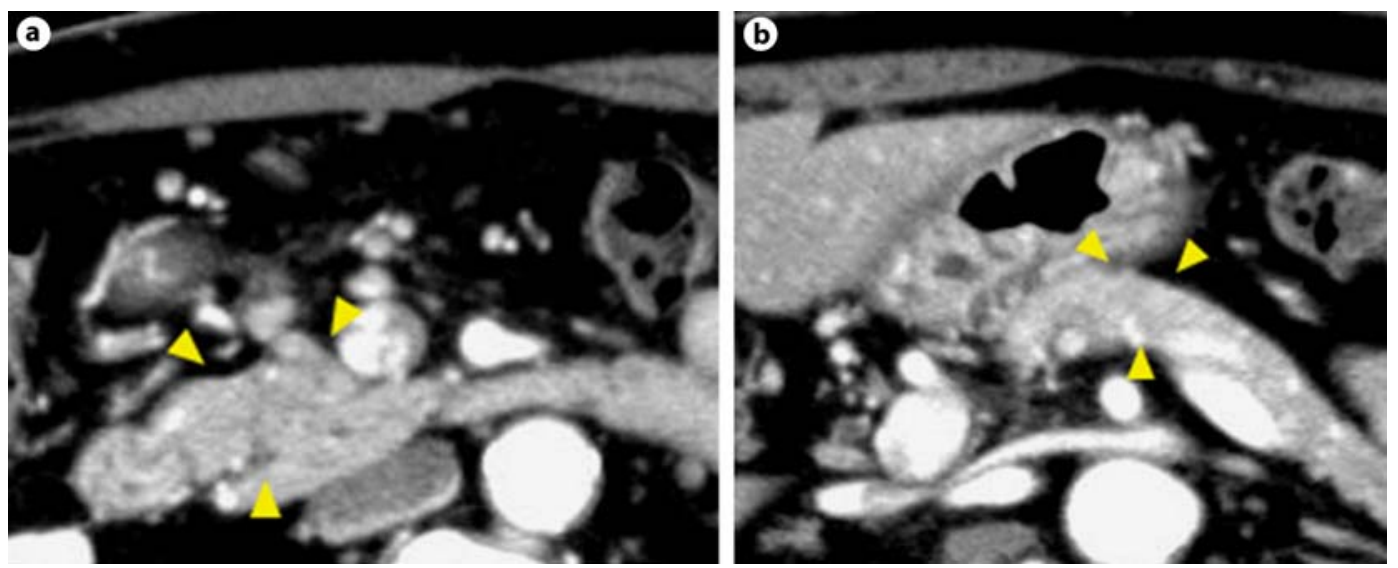

Fig. 3. The two nodules in the head (a) and body (b) had disappeared on CT.

\section{References}

$\rightarrow 1$ Finkelberg DL, et al: Autoimmune pancreatitis. N Engl J Med 2006;355:2670-2676.

2 Yoshida K, et al: Chronic pancreatitis caused by an autoimmune abnormality. Proposal of the concept of autoimmune pancreatitis. Dig Dis Sci 1995;40:1561-1568.

3 Kamisawa T, et al: Clinical difficulties in the differentiation of autoimmune pancreatitis and pancreatic carcinoma. Am J Gastroenterol 2003;98:2694-2699.

4 Matsubara T, et al: Complete obstruction of the lower common bile duct caused by autoimmune pancreatitis: is biliary reconstruction really necessary? J Hepatobiliary Pancreat Surg 2005;12:76-83.

5 Kajiwara M, et al: Autoimmune pancreatitis with multifocal lesions. J Hepatobiliary Pancreat Surg 2008;15: 449-452.

6 Sahani DV, et al: Autoimmune pancreatitis: imaging features. Radiology 2004;233:345-352.

7 Hamano $\mathrm{H}$, et al: High serum IgG4 concentrations in patients with sclerosing pancreatitis. N Engl J Med 2001;344:732-738.

8 Hirano K, Komatsu Y, Yamamoto N, et al: Pancreatic mass lesions associated with raised concentration of IgG4. Am J Gastroenterol 2004;99:2038-2040.

-9 Kamisawa T, et al: Pancreatic cancer with a high serum IgG4 concentration. World J Gastroenterol 2006;12: 6225-6228.

10 Masetti M, et al: Analysis of prognostic factors in metastatic tumors of the pancreas: a single-center experience and review of the literature. Pancreas 2010;39:135-143.

11 Metz DC, Jensen RT: Gastrointestinal neuroendocrine tumors: pancreatic endocrine tumors. Gastroenterology 2008;135:1469-1492.

12 Fujimori N, et al: Adenocarcinoma involving the whole pancreas with multiple pancreatic masses. Intern Med 2010;49:1527-1532.

13 Inoue D, et al: Autoimmune pancreatitis with multifocal mass lesions. Radiat Med 2006;24:587-591.

14 Inoue $H$, et al: A case of pancreas cancer with autoimmune pancreatitis. Pancreas 2006;33:208-209.

15 Pezzilli R, et al: Pancreatic ductal adenocarcinoma associated with autoimmune pancreatitis. Case Rep Gastroenterol 2011;5:378-385.

16 Mizuno N, et al: Histological diagnosis of autoimmune pancreatitis using EUS-guided trucut biopsy: a comparison study with EUS-FNA. J Gastroenterol 2009;44:742-750. 